\title{
Mixed signal approach for rapid prototyping of a compact smart pebble for sediment transport monitoring in river beds
}

Nihal Kularatna ${ }^{1}$, Senior Member, Chamath Wijeratne

Department of Electrical and Computer Engineering School of Engineering, University of Auckland, New Zealand

'.Email:n.kularatna@auckland.ac.nz

Abstract - Low-cost accelerometers and gyro ICs were used to develop a smart sediment particle to study the sediment transport in rivers. With strap-down MEMS, battery, a processing subsystem and memory, this self contained unit captures semi-processed data for durations up to 15 minutes. In a mixed-mode design, analog multiplier ICs with limited digital circuits transform the body frame data to a reference frame using Euler angles, with adequate accuracy despite cumulative errors. For 3D motion, up to nine sensor inputs from three orthogonal modules are coupled to a multiplexed analog processing module, and processed by a digital module for data conversion and storage. Despite the simplified mathematics used, experimental data from the proof-of-concept system provided adequate accuracy. Subsequent processing of the raw sensor data using an external PC program with smart algorithms allowed the comparison of accuracy of the mixed mode approach. The adopted mixed signal design approach helps the packaging requirements due to the specific nature of the problem with short recording durations.

\section{INTRODUCTION}

Applied research on MEMS is widely reported. Low cost MEMS, developed for automotive and consumer electronics, have been used in advanced engineering applications [1-3], including environmental and space technology. These publications show that modern accelerometers and gyro ICs are usable in strap-down inertial platforms for specialized applications, if the cumulative errors are managed with adequate accuracy. A potential application of such MEMS devices is in the development of a smart sediment particle for studies of sediment transport in rivers and elsewhere. The smart sediment particle would provide significant insight into the process of sediment entrainment [4], which would impact on diverse fields including river flooding.

\author{
Bruce Melville ${ }^{2}$ \\ Department of Civil and Environmental Engineering \\ School of Engineering, University of Auckland, New Zealand \\ 22Email:b.melville@auckland.ac.nz
}

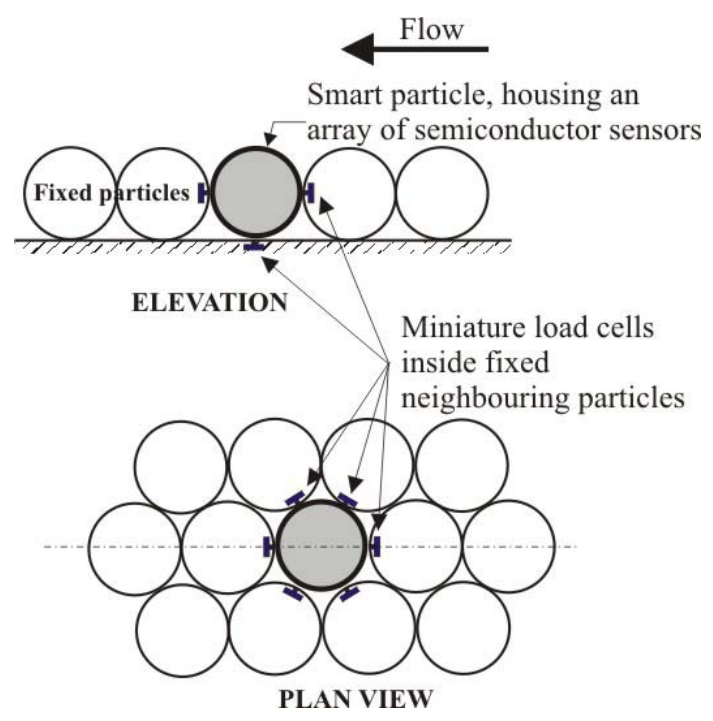

Figure 1: Test arrangement using the smart sediment particle

The granular material (sands and gravels) comprising the beds of rivers is typically in motion during periods of high river flow and stationary at other times. One of the most intriguing problems of this sediment transport process is the cause of the initial motion, i.e. the "entrainment" or pickup, of individual sediment grains. The smart sediment particle will be capable of recording its own motion in response to a range of aspects of its immediate surroundings, specifically the local water flows and the influence of the neighboring sediment particles. It will then be especially useful for elucidation of the entrainment process. Simultaneous measurement of the very complicated turbulent water flow will also be made.

Figure 1 depicts the test arrangement for use of the smart sediment particle in experiments designed to elucidate the sediment entrainment process. As shown, the smart particle will be positioned amongst a bed of similar (fixed) particles. Miniature load cells will be used to measure the inter-particle forces between the smart particle and its neighboring fixed 
particles. The measurements of the motion of the smart particle, the inter-particle forces and the flow turbulence will be used to develop a conceptual model of the entrainment process. The conceptual model will represent a most significant advance in understanding of the process of sediment transport.

In developing a "smart" pebble for this special application, the following key features were essential in addition to the basic motion-related parameter monitoring capability: (i) the physical dimensions of the pebble should be only 2 to $3 \mathrm{~cm}$ in diameter; (ii) it should operate underwater, with its specific gravity around 2.8; and (iii) it should be self contained with an energy source, memory and the ability to determine its location. Another practical problem faced by the team was to develop a proof of concept system within about 4 months, before submitting a proposal for major research funding competition. Hence, commercial off the shelf (COTS) type MEMS were used with adequately simplified inertial navigation concepts.

\section{CONCEPTS}

Inertial navigation system (INS) concepts [6] are used as the basis of this work. An INS is a system which can determine its exact location in three dimensional space, relative to a known starting location. The location is resolved by integrating the accelerations and rotations about the three axes. Three accelerometers and three gyroscopes measure the accelerations and angular rotations about the three axes, giving the particle six degrees of freedom. The reference frame consisting of three axes of the body of the pebble is known as the Body Frame. The set of sensor modules, organized in orthogonal form, measure the accelerations and gyrations with reference to the body frame. As the forces acting on the pebble need to be measured relative to a stationary frame of reference a conversion is necessary. There are several methods of performing a coordinate transformation [6]. The Euler Angle method [7] was chosen as the most appropriate method, given the processing and time constraints in this proof of concept project.

The Euler Angle method performs the coordinate transformation as three rotations about the three axes. To transform the body frame related motion parameters to the reference frame, three angular rotations, $\psi, \theta$ and $\varphi$ are performed around the $\mathrm{z}, \mathrm{y}$ and $\mathrm{x}$ axes respectively. When the three rotations are performed as per Figure 2, we get three matrices $\mathrm{C}_{1}, \mathrm{C}_{2}, \mathrm{C}_{3}$, representing the rotations as below:

Rotation $\psi$ about the reference $\mathrm{Z}$ axis,

$$
C_{1}=\left[\begin{array}{ccc}
\operatorname{Cos}(\psi) & \operatorname{Sin}(\psi) & 0 \\
-\operatorname{Sin}(\psi) & \operatorname{Cos}(\psi) & 0 \\
0 & 0 & 1
\end{array}\right]
$$

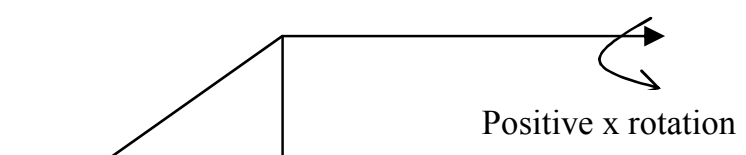

Positive $\mathrm{Z}$ rotation

Figure 2: Axis conversion from body frame to reference frame

Rotation $\theta$ about the new y-axis,

$$
C_{2}=\left[\begin{array}{ccc}
\operatorname{Cos}(\theta) & 0 & -\operatorname{Sin}(\theta) \\
0 & 1 & 0 \\
\operatorname{Sin}(\theta) & 0 & \operatorname{Cos}(\theta)
\end{array}\right]
$$

Rotation $\varphi$ about the new x-axis,

$$
C_{3}=\left|\begin{array}{ccc}
1 & 0 & 0 \\
0 & \operatorname{Cos}(\varphi) & \operatorname{Sin}(\varphi) \\
0 & -\operatorname{Sin}(\varphi) & \operatorname{Cos}(\varphi)
\end{array}\right|
$$

The three individual matrices can be combined into one complete matrix as follows:

$C_{n}^{b}=C_{3} \bullet C_{2} \bullet C_{1}$

The complete matrix $C_{n}^{b}$ is known as the rotation matrix [6].

The conversion of any vector from the body frame to the reference frame is achieved by the multiplication of the body frame vector by the transpose of the rotation matrix. This is indicated in the following:

$$
r^{n}=C_{b}^{n} \bullet r^{b}
$$

Where,

$r^{n}$ is the vector in reference frame,

$C_{b}^{n}$ is the transpose of the rotation matrix $C_{n}^{b}$, and,

$r^{b}$ is the vector in the body frame. 
For the order of rotation in Eqn (5) the transpose of the rotation matrix $C_{n}^{b}$ is as follows [6].

$C_{b}^{n}=\left[\begin{array}{ccc}\operatorname{Cos}(\theta) \operatorname{Cos}(\psi) & -\operatorname{Cos}(\varphi) \operatorname{Sin}(\psi)+\operatorname{Sin}(\varphi) \operatorname{Sin}(\theta) \operatorname{Cos}(\psi) & \operatorname{Sin}(\varphi) \operatorname{Sin}(\psi)+\operatorname{Cos}(\varphi) \operatorname{Sin}(\theta) \operatorname{Cos}(\psi) \\ \operatorname{Cos}(\theta) \operatorname{Sin}(\psi) & \operatorname{Cos}(\varphi) \operatorname{Cos}(\psi)+\operatorname{Sin}(\varphi) \operatorname{Sin}(\theta) \operatorname{Sin}(\psi) & -\operatorname{Sin}(\varphi) \operatorname{Cos}(\psi)+\operatorname{Cos}(\varphi) \operatorname{Sin}(\theta) \operatorname{Sin}(\psi) \\ -\operatorname{Sin}(\theta) & \operatorname{Sin}(\varphi) \operatorname{Cos}(\theta) & \operatorname{Cos}(\varphi) \operatorname{Cos}(\theta)\end{array}\right]$

The above rotation matrix is quite complex and a direct implementation of the same in a DSP subsystem with suitable angular inputs on sample basis is a tedious task, and certainly not feasible in a short term assignment for a student. Hence a simplified mixed signal approach based on the following special attributes of the civil engineering problem was considered.

I. The smart pebble would be in data acquisition mode for less than 30 minutes in general.

II. The pebble would not be expected to monitor motion parameters with frequency components beyond about $20 \mathrm{~Hz}$.

III. Underwater operation would be required.

IV. The pebble would need to have the correct specific gravity (about 2.65).

V. The pebble would need to be self powered, and autonomously record motion-related information for further processing later.

In developing a simplified proof of concept prototype, one important approximation used for simplifying the mathematics involved in the coordinate transformation was the case of $[\operatorname{Sin} \mathrm{x} \approx \mathrm{x}]$ and similar trigonometric relationships. In such a situation, if commercial off the shelf (COTS) accelerometers and gyroscope ICs are used to monitor instantaneous motion parameters, $r^{b}$ vector can be indicated as,

$$
r^{b}=\left[\begin{array}{c}
A_{x-b} \\
A_{y-b} \\
A_{z-b}
\end{array}\right] \ldots \ldots \ldots \ldots(7)
$$

where $A_{x-b}, A_{y-b}$ and $A_{z-b}$ are the body frame accelerations in the corresponding axes. With relatively fast sampling the angular rotations during the sampling periods being small, $\psi$, $\theta$ and $\varphi$ will be small enough to use suitable trigonometric approximations. In such a case,

$$
C_{b}^{n}=\left[\begin{array}{ccc}
1 & -\psi & \theta \\
\psi & 1 & \phi \\
-\theta & \phi & 1
\end{array}\right] \ldots \ldots \ldots .(8)
$$

This gives us the simplified relationship for the $r^{n}$ vector in the reference frame as,

$$
r^{n}=\left[\begin{array}{ccc}
1 & -\psi & \theta \\
\psi & 1 & \phi \\
-\theta & \phi & 1
\end{array}\right]\left[\begin{array}{c}
A_{x-b} \\
A_{y-b} \\
A_{z-b}
\end{array}\right] \ldots(9)
$$

For the angular rotation parameters, the following relationships were used.

$\psi=\int \omega_{z} d t, \theta=\int \omega_{y} d t, \phi=\int \omega_{x} d t$

where $\omega_{x}, \omega_{y}$ and $\omega_{z}$ are the gyrations in respective axes.

\section{IMPLEMENTAION}

In order to monitor the accelerations, COTS type accelerometers and gyroscope MEMS from Analog Devices were considered based on their sensitivity, accuracy, noise behavior and the offset, etc. ADXL202 dual axis accelerometers and ADXRS150 yaw rate gyroscopes were found suitable, with the case of ADXL202 devices providing some redundant information which could be also used in an advantageous manner. These devices were placed in three orthogonal sensor modules with suitable signal conditioning circuitry.

A mixed mode design approach was used with analog integrators, multipliers and signal conditioning circuits combined with suitable analog multiplexers and analog switches controlled by a DSP subsystem. Figure 3 indicates the overall approach and Figure 4 indicates the implementation of the coordinate transformation by a mixed mode approach. The multiplexers shown in Figure 4 are controlled by the DSP subsystem, which is used such that angles used in conversion matrix in Eqn (8) are sufficiently small for the validity of the trigonometric approximations. The multipliers shown in Figure 4 are based on Analog Devices AD 835 which is a four quadrant multiplier with a frequency capability of $250 \mathrm{MHz}$. Since dual axis accelerometers were used on the sensor modules, it was possible to read the accelerations of each axis from different sensors and estimate the average with a view to minimizing the noise and offset related imperfections. While the accelerations were averaged, the angular rotations were integrated to get the angles indicated in Eqn (8).

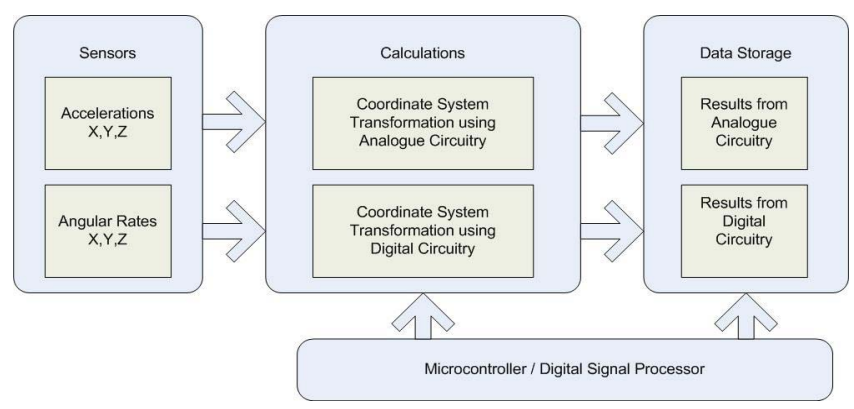

Figure 3: Simplified design concept 
The three accelerations and the three angles of rotation are fed into a series of multiplexers. The multipliers and multiplexers are organized in such a way that each term of the coordinate transformation matrix is generated by switching the multiplexers in a particular sequence. The multiplexer switching was performed by the DSP.

The DSP subsystem was based on Analog Devices ADXP 2199 development board. When the DSP performs an appropriate switching sequence, the sample and hold circuitry holds the intermediate value. Once the three terms required to calculate the accelerations in the reference frame are available at the respective $\mathrm{S} / \mathrm{H}$ circuits they are summed up by a non-inverting adder. This value is consequently sampled by the DSP and transferred to the respective ADC inputs.

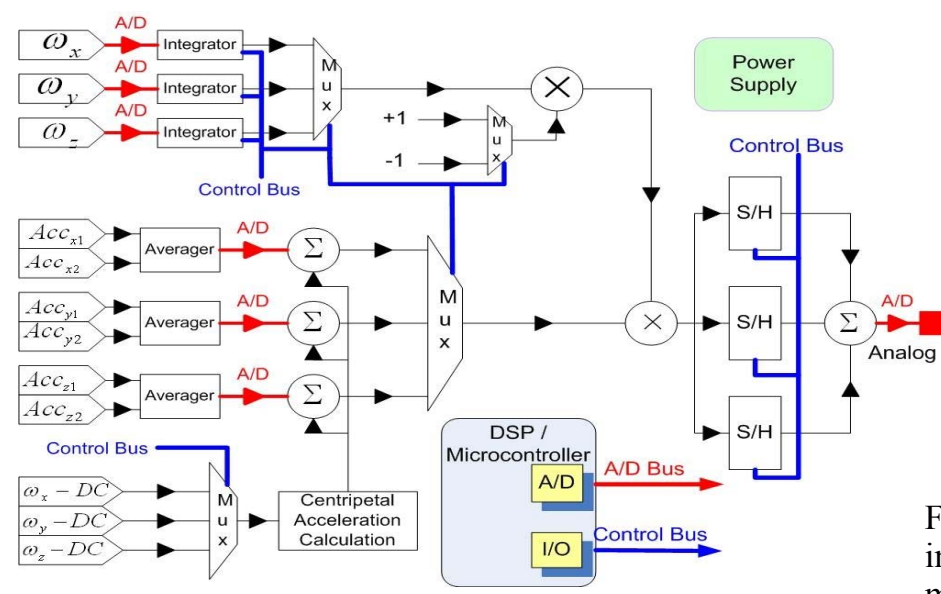

Figure 4: Implementation of analog calculation with digital control

Coordinate transformation is done at a frequency of $100 \mathrm{~Hz}$. Every time a calculation is done, the analog calculation interface is reset by the DSP to allow a fresh conversion to be done. This allows the dead reckoning process [6] to be carried out with limited accuracy without the need for a complete set of digital algorithms in the DSP subsystem. Since the civil engineering requirement could afford lower accuracies ( 2 to $5 \%$ ), this methodology is practical, given the availability of the high speed analog multiplier ICs. Figure 5 depicts the data conversion and storage strategy for the overall system, where detailed data processing could be done in a PC or a lap top.

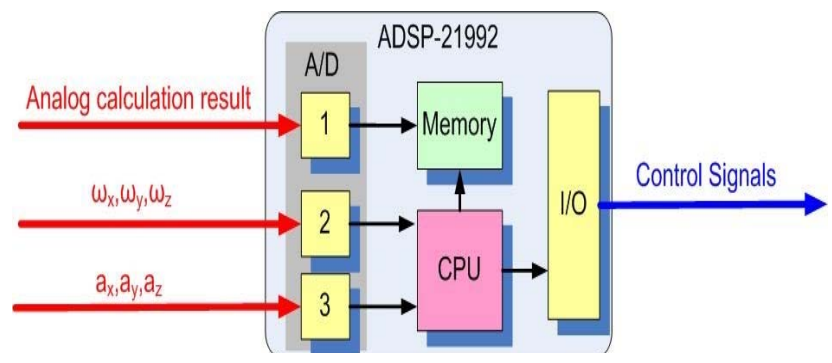

Figure 5: Data conversion and storage strategy

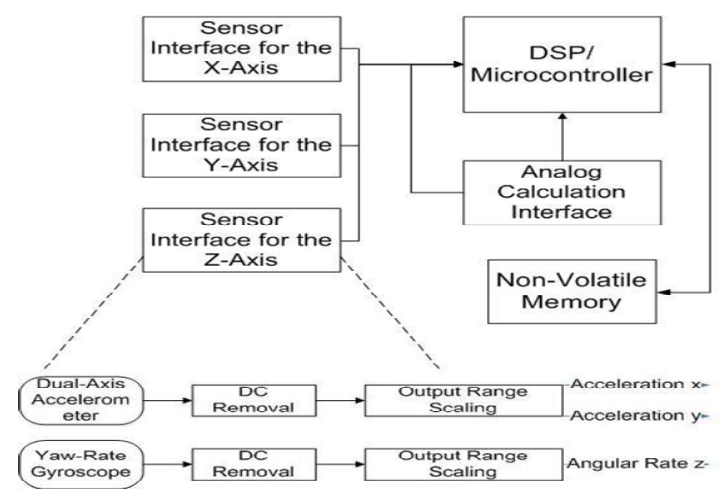

Figure 6: Overall implementation
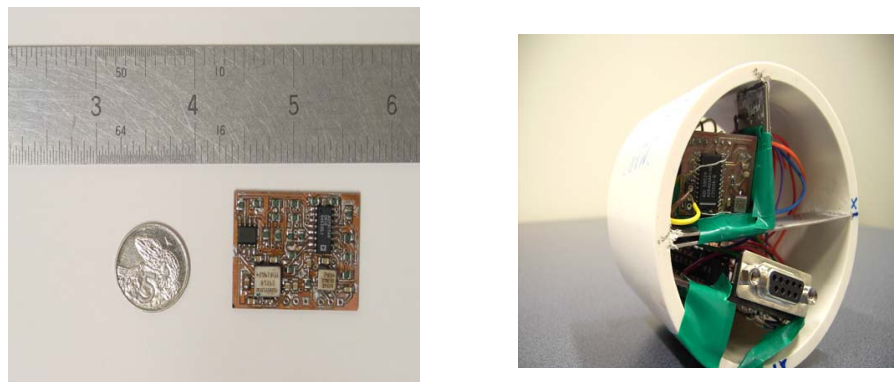

Figure 7 : Assembly (a) A sensor module (b) An early version of the pebble

Figure 6 depicts the overall block diagram of the circuitry inside the pebble with an expanded view of the sensor modules. Commencing the development with a DSP development board and configuring the overall system, the actual processing system was later reduced to an Atmel Atmega 8 processor chip with appropriate I/O circuitry and non-volatile memory. Figure 7(a) indicates the actual size of the sensor modules with one dual axis accelerometer, one gyroscope IC and the signal conditioning and filtering circuitry. Figure 7(b) indicates the total system including the lithium batteries placed in a cylindrical housing.

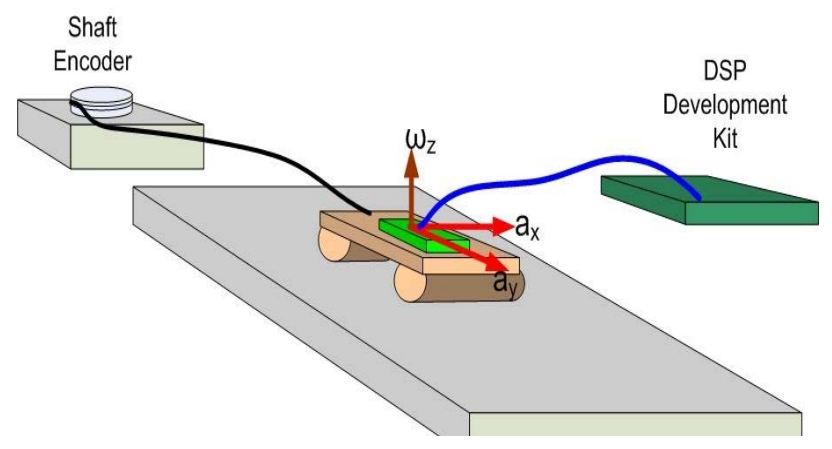

Figure 8: Calibration 

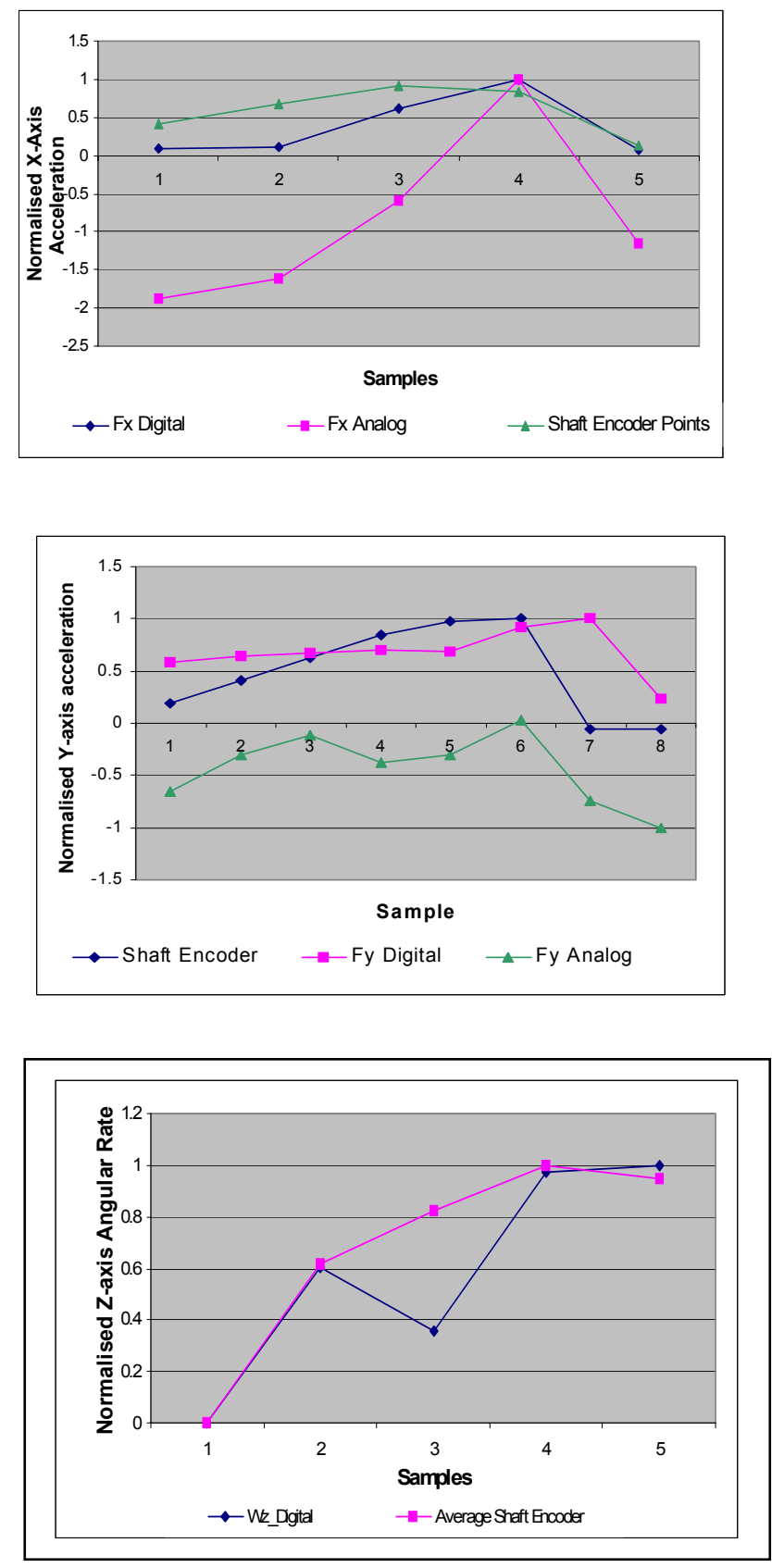

Figure 9: Comparison of analog calculations, digital readings and shaft encoder readings (a) $x$ axis (b)y axis (c) zaxis

\section{CALIBRATION AND TESTING}

In developing a system of this nature, the designer has to be aware of the overall errors contributed by many sources. Some obvious errors are the accelerometer offsets, op amp offsets and the analog calculation interface inaccuracies and offsets. The unit was mounted on a small trolley placed on a variable slope and then attached to a string coupled to digital shaft encoder for calibration. The calibration system is shown in Figure 8.

The graphs in Figure 9 indicate the measurements down the slope over an approximate period of 1.2 seconds, indicating the relationships between the analog calculation results, the digital acceleration readings and the shaft encoder measurements. Normalized motion parameters (accelerations along each axis) versus samples of these parameters indicate that there was a reasonable correlation among the sets of readings.

\section{CONCLUSION}

The project indicated that the mixed signal approach allows rapid prototyping of the proof of concept versions of a smart pebble usable for monitoring of the motion parameters. In order to save time in developing several complex mathematical algorithms for axis conversion and also to manage power consumption in an underwater situation, analog multipliers and other standard COTS type devices are employed. Further work is in progress

\section{ACKNOWLEDGMENT}

The authors are grateful to the final year project students Juyoung Sunwoo and Shuang Liew for their effort to continue the summer project work towards an early version of the smart pebble. Also we are very thankful to the technical staff member Geoff Kirby of the civil engineering laboratories for his assistance in setting up the calibration system.

\section{REFERENCES}

1. Barbour, N. \& Schmidt, G.: "Inertial sensor technology trends"; IEEE Sensors Journal, Vol. 1, No 4, Dec 2001, pp 332-339

2. Brown, T.G et el; " Strap-down micromechanical (MEMS) sensors for high-G munition applications"; IEEE Trans on Magnetics, Vol. 37, No 1, Jan 2001

3. Tieman, J. et el; " Design of a MEMS based 3-axis accelerometer smart sensor"; Proc of IEEE Sensors for Industry Conf, USA, Nov 2002, pp 19-23.

4. Coleman, S.E. and Melville, B.W. "Initiation of Bed Forms on a Flat Sand Bed", Journal of Hydraulic Engineering, ASCE, 122 (6), 301-310, June, 1996.

5. Garcia, M.H., Lopez, F. and Nino, Y. "Characterization of near-bed coherent structures in open channel flow using synchronized high-speed video and hot film measurements," Experiments in Fluids, Vol. 19, 16-28, 1995

6. Titerron, D.H. \& Weston, J.L.: " Strapdown inertial navigation technology"; IEE, 1997

7. Pio, R:"Euler angle transformations"; IEEE Transactions on Automatic Control, Volume 11, Issue 4, Oct 1966 Page(s):707 $-715$ 